\title{
Minimally invasive surgery of malignancies: time to argue the fundamental reasons for this emerging technique
}

\author{
Shengxian Yuan, Weiping Zhou \\ The Third Department of Hepatic Surgery, Naval Military Medical University Affiliated Eastern Hepatobiliary Hospital, Shanghai 200433, China \\ Correspondence to: Weiping Zhou. The Third Department of Hepatic Surgery, Naval Military Medical University Affiliated Eastern Hepatobiliary \\ Hospital, Shanghai 200433, China. Email: ehphwp@126.com. \\ Provenance and Peer Review: This article was commissioned by the Editorial Office, Hepatobiliary Surgery and Nutrition. The article did not undergo \\ external peer review.
}

Submitted Jan 20, 2020. Accepted for publication Jan 28, 2020.

doi: 10.21037/hbsn.2020.02.01

View this article at: http://dx.doi.org/10.21037/hbsn.2020.02.01

In recent years with the fast development of new surgical techniques and equipments, the indications for laparoscopic as well as robotic surgeries have continued making breakthroughs. In the case of hepatectomies, it could include total caudate lobectomy, hepatectomy for recurrences, and even combined macrovascular resection and remodeling with hepatectomy $(1,2)$. It appears that laparoscopic hepatectomy has almost no restricted area. On one hand, surgeons are enjoying pushing ceaselessly the technique to the limits, which is promising; while on the other hand, the principle of the surgery for malignancies in this new era has less been focused and discussed thus far. According to the principles of oncological surgery, instead of the incision size or length of hospital stay, the most important indicator for evaluating the success and efficacy of malignant tumor treatment is the postoperative survival time. The King of the treatment for cancers is longer survival of the patients, that has never been or should be changed, and neither to be neglected. The surgical procedure can only be improved without compromising the postoperative survival time. Any minimal invasive technology that sacrificing long-term efficacy tends to bring more physical and psychological damage to patients. In clinical practice, recurrent cancer around liver resection margin shortly after laparoscopic hepatectomy is still seen. Therefore, the attitude towards minimally invasive surgeries requires more cautious under evidencebased medicine, avoiding any aggressive technological movements.

Currently, in many medical centers, complicated laparoscopic and robotic surgeries, including laparoscopic hepatectomy, radical cholecystectomy for gall bladder cancer, peri-hilar cholangiocarcinoma resection and pancreaticoduodenectomy, are increasingly common, but there is still lack of corresponding high quality randomized controlled study to compare the radical cure rate and the postoperative survival of hepatobiliary malignant tumor between conventional and minimally invasive surgery. A recent meta-analysis from China showed that laparoscopic surgery has both a lower complication rate and a shorter time of hospital stay compared to that of open surgery. Also, the 1-, 3-, 5-year survival rates of laparoscopic surgery were relatively equivalent as that of open surgery (3). Another meta-analysis also showed that for tumors of the right posterior lobe of liver, laparoscopic surgery can achieve the same efficacy as open surgery (4). It is worth noticing that the data of both meta-analysis studies were from retrospective studies instead of randomized controlled studies with higher-level evidence, which largely reduced the validity of these conclusions. Likewise, in the field of oncological surgery, other authors subsequently reported comparative studies of open surgery versus laparoscopic surgery in early-stage cervical cancer. One study found that laparoscopic surgery was associated with a significantly lower postoperative survival than that of open surgery, thereby proposing indications of canceling laparoscopic surgery of cervical cancer $(5,6)$. These reports revealed a great controversy. Many clinicians attributed these results to the inadequacy of clinician techniques, which led to the spread of tumors or increased postoperative complications; whereas in large medical centers, clinicians have more 
patients, more opportunities to practice and more surgical experiences, so they have fewer complications and a higher chance of a radical cure. In a country like China with a large flow of patients, so far, none of the surgeons has summarized their experiences by using data to convince international peers. Although clinical experience is important, evidence matters more while evaluating the benefit of a technique. While we are introducing or promoting a new technique, we should always keep this in mind: show the proofs.

Nowadays, thousands of laparoscopic hepatectomies are performed in China each year, including any segment of liver resection. However, as a surgeon who performed the first laparoscopic hepatectomy in China, I must say that most surgeons are satisfied by "I can do". They are glad to show surgical techniques like surgical approaches, bleeding control, or intrahepatic vascular dissection, but they lack summaries about the key differences between open and laparoscopic surgery, like complications, postoperative recurrence rate, and more importantly survival rate. Many of the surgeons have not reached the altitude of "why I do it". Compared with the technical level of "I can do", the latter is a decision-making behavior that requires evidence. The prognosis of liver tumors and the difficulties of laparoscopic surgery are affected by tumor size, location, vascular invasion, cirrhosis, and so on. How to define indications of minimally invasive surgery, that is, under what circumstances that laparoscopic or robotic hepatectomy does not have a worse overall survival rate compared to that of conventional surgery, should be performed in randomized controlled studies according to different tumor characteristics. This step requires numerous clinical trials to verify, which contains a lot of opportunities for those hepatobiliary surgeons. Therefore, the problem that Chinese clinicians need to solve is to analyze and summarize in time based on exploring new technologies and methods, to compare with traditional methods and to find their shortcomings and to improve them. Carrying out higher-level evidence-based randomized controlled studies to obtain more objective and reliable data to guide future clinical works, providing foundations for updating or drafting new guidelines, the surgical level can be truly improved. For example, currently, many international guidelines (such as the NCCN Guidelines) still do not recommend performing laparoscopic surgery in radical cholecystectomy for gall bladder cancer. Laparoscopy is only recommended for exploration because there is no evidence supporting that laparoscopic surgery can achieve a radical effect equivalent to that of open surgery. In fact, this provides a good opportunity for us. As long as we design and conduct studies strictly and carefully, we can obtain evidence in a relatively short period of time, thus letting more of our voices be heard in the field. The advantage of surgeons in a country like China is that they have a large amount of patients. The studies can include enough quantity of cases in a short period of time. However, the problem is still existed that each surgical center only concerns about their own studies. To coordinate different surgical centers, to achieve data opening and resource sharing, and to perform standardized, uniformly highquality randomized controlled studies requires coordination on a national or international basis.

\section{Acknowledgments}

Funding: None.

\section{Footnote}

Conflicts of Interest: The authors have no conflicts of interest to declare.

Ethical Statement: The authors are accountable for all aspects of the work in ensuring that questions related to the accuracy or integrity of any part of the work are appropriately investigated and resolved.

Open Access Statement: This is an Open Access article distributed in accordance with the Creative Commons Attribution-NonCommercial-NoDerivs 4.0 International License (CC BY-NC-ND 4.0), which permits the noncommercial replication and distribution of the article with the strict proviso that no changes or edits are made and the original work is properly cited (including links to both the formal publication through the relevant DOI and the license). See: https://creativecommons.org/licenses/by-nc$\mathrm{nd} / 4.0 \%$.

\section{References}

1. Vega EA, Nicolaescu DC, Salehi O, et al. Laparoscopic Segment 1 with Partial IVC Resection in Advanced Cirrhosis: How to Do It Safely. Ann Surg Oncol 2020;27:1143-44.

2. Onoe T, Yamaguchi M, Irei T, et al. Feasibility and efficacy of repeat laparoscopic liver resection for recurrent hepatocellular carcinoma. Surg Endosc 2019. [Epub ahead 
of print].

3. Wang ZY, Chen QL, Sun LL, et al. Laparoscopic versus open major liver resection for hepatocellular carcinoma: systematic review and meta-analysis of comparative cohort studies. BMC Cancer 2019;19:1047.

4. Zheng H, Huang SG, Qin SM, et al. Comparison of laparoscopic versus open liver resection for lesions located in posterosuperior segments: a meta-analysis of short-term and oncological outcomes. Surg Endosc 2019;33:3910-8.

5. Melamed A, Margul DJ, Chen L, et al. Survival after Minimally Invasive Radical Hysterectomy for Early-Stage Cervical Cancer. N Engl J Med 2018;379:1905-14 .

6. Ramirez PT, Frumovitz M, Pareja R, et al. Minimally Invasive versus Abdominal Radical Hysterectomy for Cervical Cancer. N Engl J Med 2018;379:1895-904.

Cite this article as: Yuan S, Zhou W. Minimally invasive surgery of malignancies: time to argue the fundamental reasons for this emerging technique. HepatoBiliary Surg Nutr 2020;9(2):195-197. doi: 10.21037/hbsn.2020.02.01 